\title{
Optimizing the operating time of wireless sensor network
}

\author{
Thanh Tung Nguyen ${ }^{1 *}$ and Van Duc Nguyen ${ }^{2}$
}

\begin{abstract}
A difficult constraint in the design of wireless sensor networks (WSNs) is the limited energy resource of the batteries of the sensors. This limited resource restricts the operating time that WSNs can function in their applications. Routing protocols play a major part in the energy efficiency of WSNs because data communication dissipates most of the energy resource of the networks. There are many energy-efficient cluster-based routing protocols to deliver data from sensors to a base station. All of these cluster-based algorithms are heuristic. The significant benefit of heuristic algorithms is that they are usually very simple and can be utilized for the optimization of large sensor networks. However, heuristic algorithms do not guarantee optimal solutions. This article presents an analytical model to achieve the optimal solutions for the cluster-based routing protocols in WSNs.
\end{abstract}

Keywords: Sensor networks, Routing, Cluster networks, Battery, Linear programming, Optimization.

\section{Introduction}

There is a common problem in energy efficiency considerations in wireless sensor networks (WSNs): maximizing the amount of data sent from all sensor nodes to the base station (BS) until the first sensor node is out of battery. In sensor networks, sensors send data to each BS periodically during each fixed amount of time. Thus, the problem is the same as maximizing network operation lifetime until the first sensor node run out of battery. Numerous studies have been done on the energy efficiency using cluster-based routing in WSNs [1-5]. Cluster-based routing was originally used to solve the scalability problems and resources-efficient communication problems in wire-line and wireless networks $[6,7]$. The method can also be used to perform energyefficient routing in WSNs. In the cluster-based routing, nodes cooperate to send sensing data to a BS. In this routing, a network is organized into clusters and nodes play different roles in the network. A node with higher remaining energy can be elected as the cluster head $(\mathrm{CH})$ of each cluster. This node is responsible to receive data from its members in the cluster and to send the data to the BS.

\footnotetext{
* Correspondence: tungnt@isvnu.vn

${ }^{1}$ International School, Vietnam National University, 144 Xuan Thuy, Cau Giay, Hanoi, Vietnam

Full list of author information is available at the end of the article
}

However, all of the above-mentioned cluster-based routing work is heuristic. The real benefit of heuristic algorithms is that they are usually very simple and can be used for the optimization of large sensor networks. However, in general, heuristic algorithms do not guarantee optimal solutions.

In this article, an analytical model is used to obtain the optimal solutions for the above clustering lifetime problem. The basic idea is to formulate the problem as an integer linear programming (ILP) problem and to utilize ILP solvers [8] to compute the optimal solutions. These solutions are employed to evaluate the performance of previous heuristic algorithms. These analytical models are used to formulate the system lifetime problem into a simpler problem, find the optimum solution for the system lifetime problem, and evaluate the performance of heuristic models.

This article is organized as follow. The following section summarizes previous work in energy efficiency using cluster-based routing. Then, an analytical model of the cluster-based routing is developed. The model is first implemented by an analysis of a simple network with one cluster. After that, the analysis is extended for more complex cases of multiple clusters. A new heuristic cluster-based routing is also proposed. Finally, the simulation results of the analytical model, old heuristic solutions, and the new ones are presented and discussed.

\section{空 Springer}




\section{Previous work in energy efficiency using cluster-based routing}

In a cluster-based routing, higher remaining energy nodes can gather data from low ones, perform data aggregation, and send the data to a BS. Nodes in networks are grouped into clusters, and nodes that have higher remaining energy are elected as the CHs. In each cluster, the nominated $\mathrm{CH}$ node receives and aggregates data from all sensor nodes in the cluster. Usually, the sizes of the data of all sensors are the same and the aggregated data at the $\mathrm{CH}$ node has the same size with the data of every sensor in the cluster. As the data are aggregated in the $\mathrm{CH}$ node before reaching a $\mathrm{BS}$, this technique reduces the amount of information sent to the distant BS, hence saves energy. For example, if each sensor in the cluster sends a message of 100 bits to the $\mathrm{CH}$ node, then the $\mathrm{CH}$ node sends the aggregated message of 100 bits to the BS. Details are given in $[2,6,9]$. As shown in Figure 1, all nodes in Cluster 1 send data to the $\mathrm{CH}$. The node aggregates the data with its own data and sends the final data to the BS.

In sensor applications, every sensor node sends data periodically to its BS. Initially, every node starts with the initialized battery storage. A round of data transmission is defined as the duration of time to send a unit of data to the BS. At the end of each round, every sensor node loses an amount of energy which is used to send a unit of data to the BS. The lifetime of sensor networks is

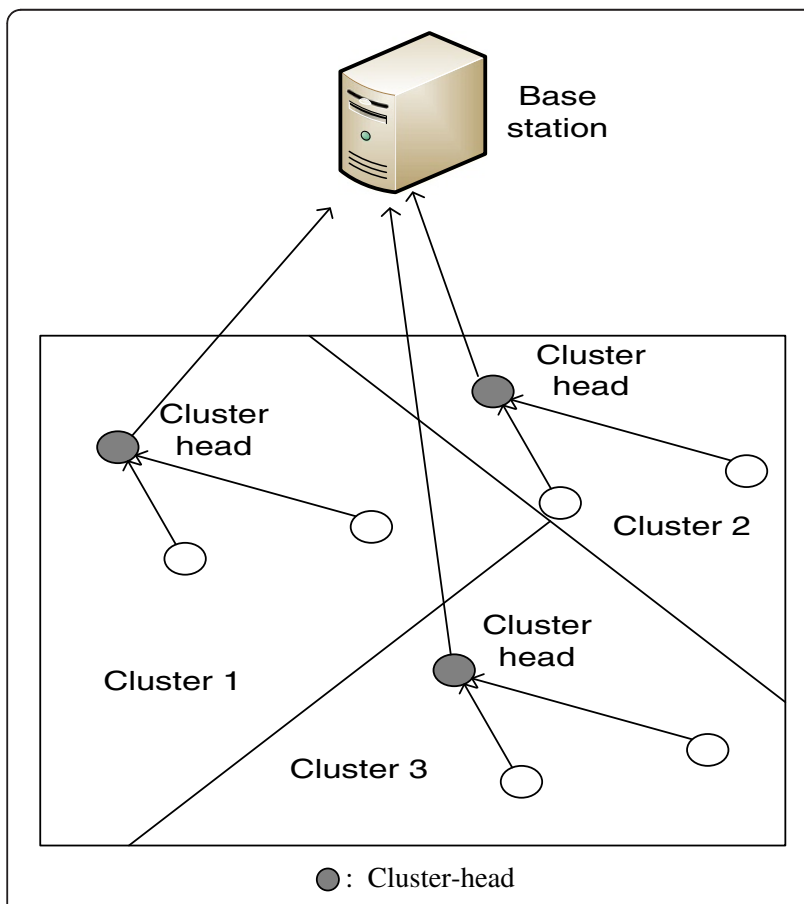

Figure 1 In cluster-based routing, networks are divided into clusters, in which a node is elected as the $\mathrm{CH}$ for each cluster. defined as the total number of rounds sending data to the BS until the first node is off.

Heinzelman et al. [1,2] proposed a Low-Energy Adaptive Clustering Hierarchy (LEACH). In LEACH, the operation of the protocol is divided into rounds. Each round consists of the setup and the transmission phase. In the setup phase, the network is divided into clusters and nodes negotiate to nominate $\mathrm{CHs}$ for the round. In more details, during the setup phase, a predetermined fraction of nodes, $p$, elect themselves as $\mathrm{CHs}$ as follows. A node picks a random number, $r$, between 0 and 1 .

\section{If $(r<T(n))$ then}

The node becomes a $\mathrm{CH}$ for the current round else

The node remains a non- $\mathrm{CH}$ node

where $T$ is a threshold value given by:

$$
T(n)=p: n \in G,
$$

where $G$ is the set of nodes that are involved in the $\mathrm{CH}$ election. The selected $\mathrm{CHs}$ for the round advertise themselves as the round's new $\mathrm{CHs}$ to the rest of the nodes in the network. All the non- $\mathrm{CH}$ nodes decide on the cluster to which they want to belong to. The decision is based on the distance to the closest $\mathrm{CH}$.

In the transmission phase of $\mathrm{LEACH}$, the elected $\mathrm{CH}$ collects all the data from nodes in its cluster, aggregates these data, and forwards them to a BS. In the next rounds, the process is repeated and $\mathrm{CH}$ positions are reallocated among all nodes in the network to extend the network lifetime.

For examples, as can be seen from Figure 2, the role of $\mathrm{CH}$ for Zone 1 is moved from Node 2 to Node 1 and the role of $\mathrm{CH}$ for Zone 2 is moved from Node 4 to Node 3 in the next round of data transmission. Therefore, the energy dissipation of these nodes during the network operation is balanced.

The LEACH protocol ensures that every node can become a $\mathrm{CH}$ exactly once within $1 / p$ rounds. This will not give the optimum network lifetime, as sensor nodes that are far away from the BS will consume more energy than closer nodes to send data to the BS. Therefore, nodes, which are close to BS, need to become $\mathrm{CHs}$ more frequently than other nodes.

There are some LEACH variants to address the above issues in LEACH protocol [3,10-13]. Saha Misra et al. [3] proposed the energy enhanced-efficient adaptive clustering protocol for distributed sensor networks. $\mathrm{CHs}$ can be formed based on the residual energy of each node. The residual energy is calculated for every node after each round of transmission. Every node transmits a code containing the information about its residual 


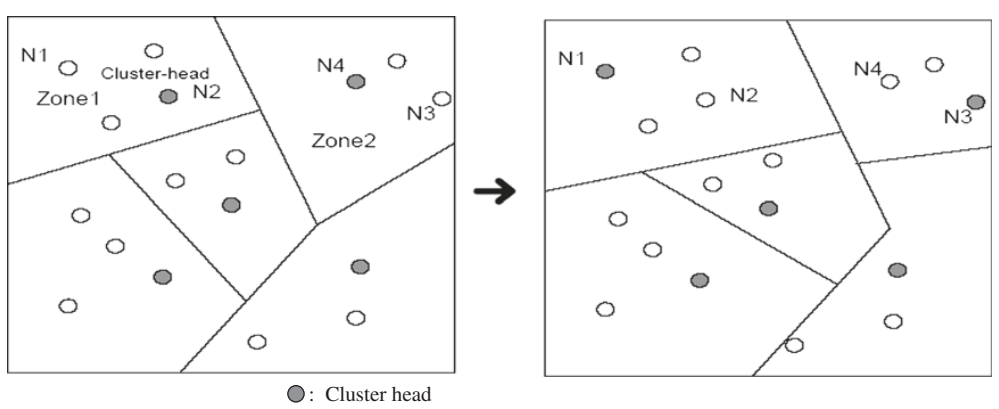

Figure $\mathbf{2} \mathrm{CHs}$ are reallocated in different rounds of transmission.

energy and its identification. If this residual energy is more than the ones of all other nodes in the same sub-area, then the node is the $\mathrm{CH}$ for that round in this sub-area. Otherwise, it can detect the node that has the maximum residual energy and elects this node as the $\mathrm{CH}$.

A different approach was used by the authors of $[4,5]$ who add the current energy information of sensor nodes into Equation (1).

$$
T(n)=\frac{p}{1-p(r \bmod (1 / p))} \times \frac{E_{\text {current }}}{E_{\text {initial }}}, n \in G,
$$

where $E_{\text {current }}$ is the current energy of Node $n$ and $E_{\text {initial }}$ is the initial energy of the node.

If $(r<T(n))$ then

The node becomes a $\mathrm{CH}$ for the current round

else

The node remains a non- $\mathrm{CH}$ node

Simulation results showed that the lifetime of the network with the scheme is improved 30\% compared with the LEACH algorithm under the same experiments for LEACH.

After the design of LEACH protocol, these authors further proposed a new centralized version called LEACH_C in [2]. Unlike LEACH, LEACH_C utilizes the BS for creating clusters. During the setup phase, the BS receives the information about the location and the energy level of each node in the network. Using this information, the BS decides the number of $\mathrm{CHs}$ and configures the network into clusters. To accomplish this, the BS computes the average energy of nodes in the network, and nodes that have energy storage below this average cannot become $\mathrm{CHs}$ for the next round. From the remaining $\mathrm{CH}$ nodes, the $\mathrm{BS}$ uses the simulated annealing (SA) algorithm to find the $k$ optimal CHs. The selection problem is an NP-hard problem $[14,15]$. The solution attempts to minimize the total energy required for non- $\mathrm{CH}$ nodes in sending data to the corresponding $\mathrm{CHs}$. As soon as the $\mathrm{CHs}$ are found, the $\mathrm{BS}$ broadcasts a message that contains a list of $\mathrm{CHs}$ for all sensors. If a node CH's ID matches its own ID, the node becomes a $\mathrm{CH}$. Otherwise, the node determines its TDMA slot for its data transmission from the broadcast message and turns off its radio until the transmission phase. The transmission phase of LEACH_C is identical to that of LEACH. Under the same experimental settings, LEACH_C improves LEACH from 30 to $40 \%$.

Besides cluster-based routings [10-13], there is also a chain-based one. Lindsey and Raghavendra [16] proposed one type of chain-based protocol called powerefficient gathering in sensor information systems (PEGASIS), which is near optimal for gathering data in sensor networks. PEGASIS forms a chain among sensor nodes so that each node will receive data from a near neighboring node and transmit data to another near neighbor. Gathered data move from a sensor node to the nearest neighbor, are aggregated with the neighbor's data, and eventually reach a determined $\mathrm{CH}$ before finally being transmitted to the BS. Figure 3 illustrates the ideas of the PEGASIS protocol. In this round of data transmission, Node 3 is elected as the $\mathrm{CH}$. Node 5 transmits data to Node 4, and Node 4 fuses the data with its own data and transmits the fused data to Node 3. Similarly, Node 1 transmits data to Node 2, and Node 2 transmits the fused data to Node 3. Finally, Node 3 fuses the data of the other nodes with its own data and transmits the final fused data to the BS. The data fusion function can be any function, e.g., minima, maxima, and average, depending on specific applications. Nodes take turns equally to be the $\mathrm{CH}$ so that the energy spent by each node is balanced. In other words, each node becomes a $\mathrm{CH}$ once for every $n$ rounds of data transmission, where $n$ is the number of sensor nodes.

The comparison between the chain-based routings and cluster-based routings were done extensively in [9] and this is not mentioned here as this article only focuses on cluster-based routing.

In the next section, an analytical model is presented to achieve the optimal solutions for the frequency of $\mathrm{CHs}$ of sensor nodes. The basic idea is to formulate the problem as an ILP problem and to utilize ILP solvers [8] to 


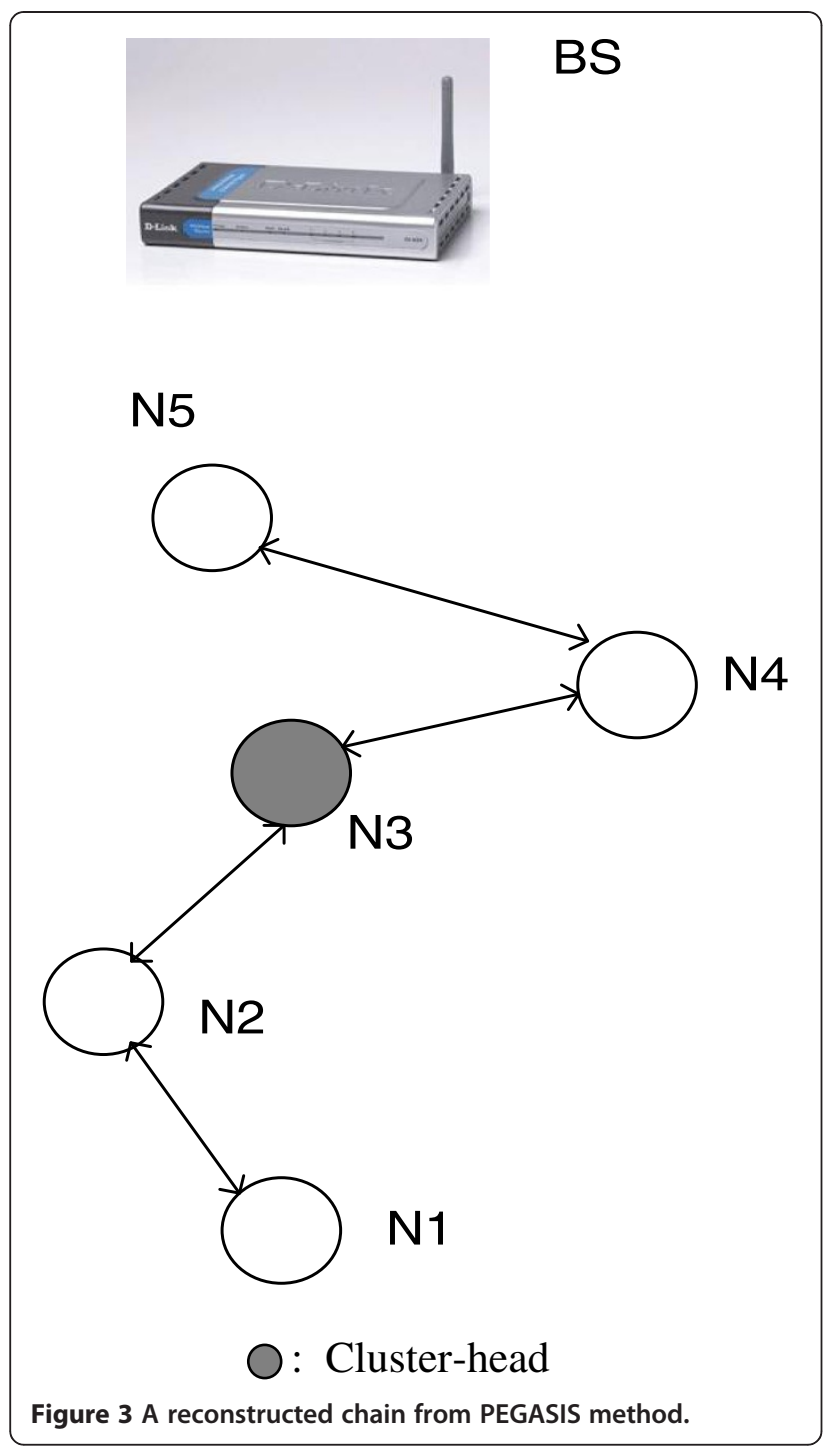

compute the optimal solutions. These solutions are employed to evaluate the performance of previous heuristic algorithms.

\section{Analytical model for optimizing the lifetime of sensor network with one $\mathrm{CH}$}

In order to minimize the complexities of the clustering problem, the wireless radio energy dissipation model is not used. This assumption does not change the validation of any simulation result. A very simple energy usage model is given as

$$
E(S)=\alpha d^{2}, E(D)=0, \text { for } \alpha>0
$$

where $S$ denotes a source node, $D$ denotes a destination node, $E(S)$ is the energy usage of node $S$, and dis the distance from $S$ to $D$. This formula states that the energy required to transmit a unit of data is proportional to the square of the distance to a destination, and there is no energy spent at the destination. In this section, $\alpha$ is set to 1 .

Let us analyze a very simple network to establish a general method that can be applied for any complicated problem. Figure 4 shows a simple network topology in which there are five nodes that lie on a line. The nodes are located equally from position 0 to position $80 \mathrm{~m}$ and the BS is located on the position $175 \mathrm{~m}$. In sensor applications, every sensor node sends data periodically to the BS. A round of data transmission is defined as the duration of time to send a unit of data to the BS. Therefore, the lifetime of sensor networks is defined as the total number of rounds of sending data to the BS until the first node is off. It is assumed that every node starts with the equal initial battery storage of 500,000 units. The problem is maximizing the total the number of rounds of sending data to the BS until the first sensor node runs out of battery.

In each round of operation, every node must transmit a unit of data to the BS. It is also assumed that only one node acts as the $\mathrm{CH}$ in each round of transmission and the role is reallocated among all nodes so the system lifetime is maximized. The analytical model needs to compute the optimal usage of nodes as $\mathrm{CHs}$ under the battery constraint of every sensor.

Let us denote $x_{j}, \forall j \in[1 \ldots 5]$ to be the number of rounds, which Node $j$ becomes a $\mathrm{CH}$ and $c_{j}^{i}$ be the energy consumption of Node $i$, to deliver a unit of data in each round, when Node $j$ becomes a $\mathrm{CH}, \forall i, j \in[1 \ldots 5]$. As there are five nodes and only one $\mathrm{CH}$, there are five possible choices for the $\mathrm{CH}$ in each round and there are also five energy usages for these five sensor nodes, respectively. This is shown in Table 1 . For example, the energy dissipation of Node 1 when Node 5 becomes a

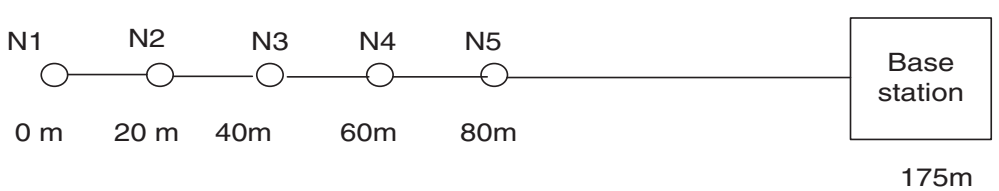

Figure 4 A simple network topology of five nodes on a line. 
Table 1 The energy dissipated $c_{j}^{i}$ (units) per round of node $i$ when node $\boldsymbol{j}$ becomes a $\mathrm{CH}$

\begin{tabular}{llllll}
\hline & Node 1 & Node 2 & Node 3 & Node 4 & Node 5 \\
\hline $\mathrm{CH} 1$ & $\mathbf{3 0 6 2 5}$ & 400 & 1600 & 3600 & 6400 \\
$\mathrm{CH} 2$ & 400 & $\mathbf{2 4 0 2 5}$ & 400 & 1600 & 3600 \\
$\mathrm{CH} 3$ & 1600 & 400 & $\mathbf{1 8 2 2 5}$ & 400 & 1600 \\
$\mathrm{CH} 4$ & 3600 & 1600 & 400 & $\mathbf{1 3 2 2 5}$ & 400 \\
$\mathrm{CH} 5$ & 6400 & 3600 & 1600 & 400 & $\mathbf{9 0 2 5}$ \\
\hline
\end{tabular}

$\mathrm{CH}, c_{5}^{1}$ is $(80-0)^{2}=6400$, the energy dissipation of Node 1 when Node 1 becomes a $\mathrm{CH}, c_{1}^{1}$ is $(175-0)^{2}=$ 30625. The optimum number of transmission rounds (or system lifetime) for the network is written as the following ILP problem.

Maximize: $\sum_{j=1}^{5} x_{j}$

Subject to:

$$
\sum_{j=1}^{5} c_{j}^{i} x_{j} \leq E_{i}: \forall i \in[1 \ldots 5] x_{j} \in Z^{+}: \forall j \in[1 \ldots 5]
$$

where $E_{i}$ is the initial battery storage of node $i$. Formulation (3) states that the total number of rounds must satisfy the battery storage constraint of every sensor node.

Table 2 shows the optimum result obtained from (3) when the battery capacity increases from 125,000 to 50 million units. When the battery size is large enough (greater than 1 million units), the number of rounds that each node becomes a $\mathrm{CH}$ increases almost linearly with the battery capacity (e.g., the number of rounds of each node is nearly doubled when the battery capacity is increased from 1 to 2 million).

\section{Simplification of formulation (3)}

Formulation (3) can be converted to a linear programming (LP) formulation as given below:

$$
\text { Maximize: } \sum_{j=1}^{n} x_{j}
$$

Table 2 The number of rounds that each node $i$ is a $\mathrm{CH}$ over the number of initial battery $E$ (units) of each node

\begin{tabular}{llllll}
\hline $\boldsymbol{E}$ & Node $\mathbf{1}$ & Node $\mathbf{2}$ & Node $\mathbf{3}$ & Node 4 & Node 5 \\
\hline 125,000 & 0 & 2 & 5 & 8 & 11 \\
250,000 & 0 & 5 & 11 & 17 & 13 \\
500,000 & 1 & 11 & 22 & 34 & 44 \\
1000,000 & 3 & 23 & 44 & 68 & 88 \\
2000,000 & 7 & 46 & 89 & 135 & 176 \\
50 millions & 180 & 1155 & 2241 & 3391 & 4404 \\
\hline
\end{tabular}

Subject to:

$$
\sum_{j=1}^{n} c_{j}^{i} x_{j} \leq E_{i}: \forall i \in[1 \ldots n] x_{j} \geq 0: \forall j \in[1 \ldots n]
$$

where the condition of variables being integers is removed. There are two cases to use the formulation to obtain the optimization solutions:

(1) $E_{i} \rightarrow \infty$ then the solution of (4) becomes the solution of (3)

(2) $E_{i} \neq \infty$ then the solution of (4) is the approximation of the solution of (3)

Formulation (4) can remove the NP-hard characteristic of the ILP formulation (3). Therefore, the optimization solution can be solved by the simplex method $[8,9]$. In the next section, we will verify the solutions obtained from both formulations. A simple network topology of 11 nodes is given in Figure 5. All nodes are located equally on the line. The nodes are located equally from position 0 to position $100 \mathrm{~m}$ (separated each $10 \mathrm{~m}$ ) and the BS is located on the position $175 \mathrm{~m}$.

In the simulation, each node starts with an equal amount of initial energy of 500 million units. The lifetime problem for the network is first formulated as an ILP problem using (3). Then the LP formulation as in (4) is used to calculate the approximate solutions. Table 3 shows that the solutions given by both methods are almost identical. Therefore, the formulation of (4) can be an approximating solution of (3). Also, Nodes 10 and 11 never become a $\mathrm{CH}$ as they are too far from other nodes. Node 1 will never become a $\mathrm{CH}$ as it is too far from the BS.

\section{Analytical model for optimizing the lifetime of sensor network with multiple $\mathrm{CH}$}

The previous section assumes a very simple case when there is only one $\mathrm{CH}$. It is obvious that for the simple network of Figure 4, too many CHs will drain the energy of all sensor nodes very quickly as the nodes have to send data to the distant BS. This is not true for the other network topologies. The network considered in the analysis section has 20 nodes. The network topology is given in Figure 6. All nodes are located equally on the two lines.

For the network, one $\mathrm{CH}$ could not be enough, as other non- $\mathrm{CH}$ nodes would consume energy significantly to deliver a unit of data to the $\mathrm{CH}$ in each round. Table 4 shows the performance of the network with a variable number of clusters. The simulation result shows that two $\mathrm{CHs}$ will minimize the total energy consumption to send data to the BS. 


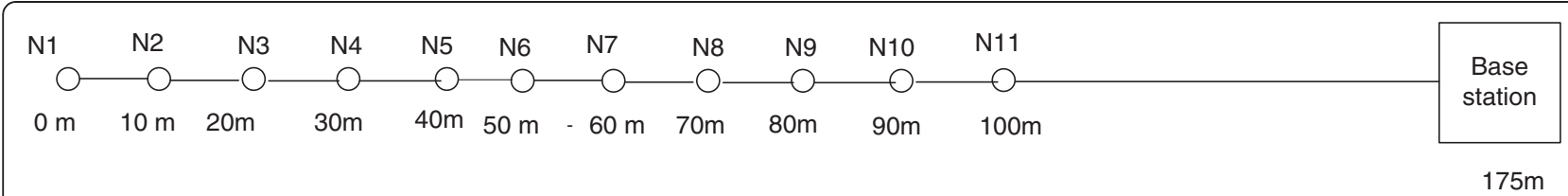

Figure 5 A simple topology of 11 nodes on a line.

When the number of $\mathrm{CHs}$ is more than one, it is much more complicated to obtain optimum solutions. The number of possible combinations of $\mathrm{CHs}$ is $O\left(n^{k}\right)$, where $n$ is the number of sensor nodes and $k$ is the number of $\mathrm{CHs}$. Furthermore, with a selected solution of $\mathrm{CHs}$, each sensor has $k$ choices to select its $\mathrm{CH}$. Therefore, the method of finding the optimum solution includes two optimization processes: optimization of the position of $\mathrm{CHs}$ and optimization of gathering traffic to the $\mathrm{CHs}$.

In order to design an analytical model for complex cases with multiple $\mathrm{CH}$ in sensor networks, Theorem 1 is stated and proved.

Theorem 1: Consider two ILP problems with the same objective function and the same variables, if the set of coefficients of ILP problem 2 is smaller than the set of coefficients of ILP problem 1, respectively, for all of these coefficients, then the optimal solution of Problem 2 is higher than that of Problem 1.

Consider two ILP problems:

Problem 1:

Maximize: $\sum_{j=1}^{n} x_{j}$

Table 3 The number of rounds each node $i$ becomes a $\mathrm{CH}$ solved by formulations (2) and (3)

\begin{tabular}{lll}
\hline Node $\boldsymbol{i}$ & Formulation (2) & Formulation (3) \\
\hline 1 & 0 & 0 \\
2 & 569 & 569.6 \\
3 & 1152 & 1152.3 \\
4 & 1737 & 1737.5 \\
5 & 2307 & 2307.2 \\
6 & 2831 & 2831.2 \\
7 & 3258 & 3258.7 \\
8 & 3503 & 3503.3 \\
9 & 1290 & 1289.1 \\
10 & 0 & 0 \\
11 & 0 & 0 \\
Total & 16647 & 16646 \\
\hline
\end{tabular}

Subject to:

$$
\sum_{j=1}^{n} c_{j}^{i} x_{j} \leq E_{i}: \forall i \in[1 \ldots m] x_{j} \in Z^{+}: \forall j \in[1 \ldots n]
$$

Problem 2:

Maximize: $\sum_{j=1}^{n} x_{j}$

Subject to:

$$
\sum_{j=1}^{n} c_{j}^{\prime i} x_{j} \leq E_{i}: \forall i \in[1 \ldots m] x_{j} \in Z^{+}: \forall j \in[1 \ldots n]
$$

Definition: $O_{1}$ is the optimal solution of Problem (5). $\mathrm{O}_{2}$ is the optimal solution of Problem (6)

$$
\text { If } c_{j}^{i i} \leq c_{j}^{i} \forall i \in[1 \ldots m], \forall j \in[1 \ldots n] \text {, then } O_{2} \geq O_{1}
$$

Proof: Since $c^{\prime}{ }_{j}^{i} \leq c_{j}^{i} \forall i \in[1 \ldots m], \forall j \in[1 \ldots n]$ and $O_{1}$ is the optimal solution of Problem 1, then $O_{1}$ is a feasible solution of Problem 2 because $O_{1}$ satisfy all constraints of (6). Since $\mathrm{O}_{2}$ is the optimal solution of Problem 2, $\mathrm{O}_{2} \geq \mathrm{O}_{1}$

To illustrate Theorem 1, let us consider two simple ILP problems:

Simple problem 1:

Maximize $x_{1}+x_{2}$

Subject to:

$$
\frac{2 x_{1}+3 x_{2} \leq 20}{3 x_{1}+4 x_{2} \leq 20} x_{1}, x_{2} \in Z^{+}
$$

Simple problem 2:

Maximize $x_{1}+x_{2}$

Subject to:

$$
\frac{x_{1}+2.5 x_{2} \leq 20}{2.5 x_{1}+3.5 x_{2} \leq 20} x_{1}, x_{2} \in Z^{+}
$$

Applying Theorem 1 for two simple problems (1) and (2), as the coefficients of the constraint functions (7) are all higher than those of (8) respectively, the optimal solution 


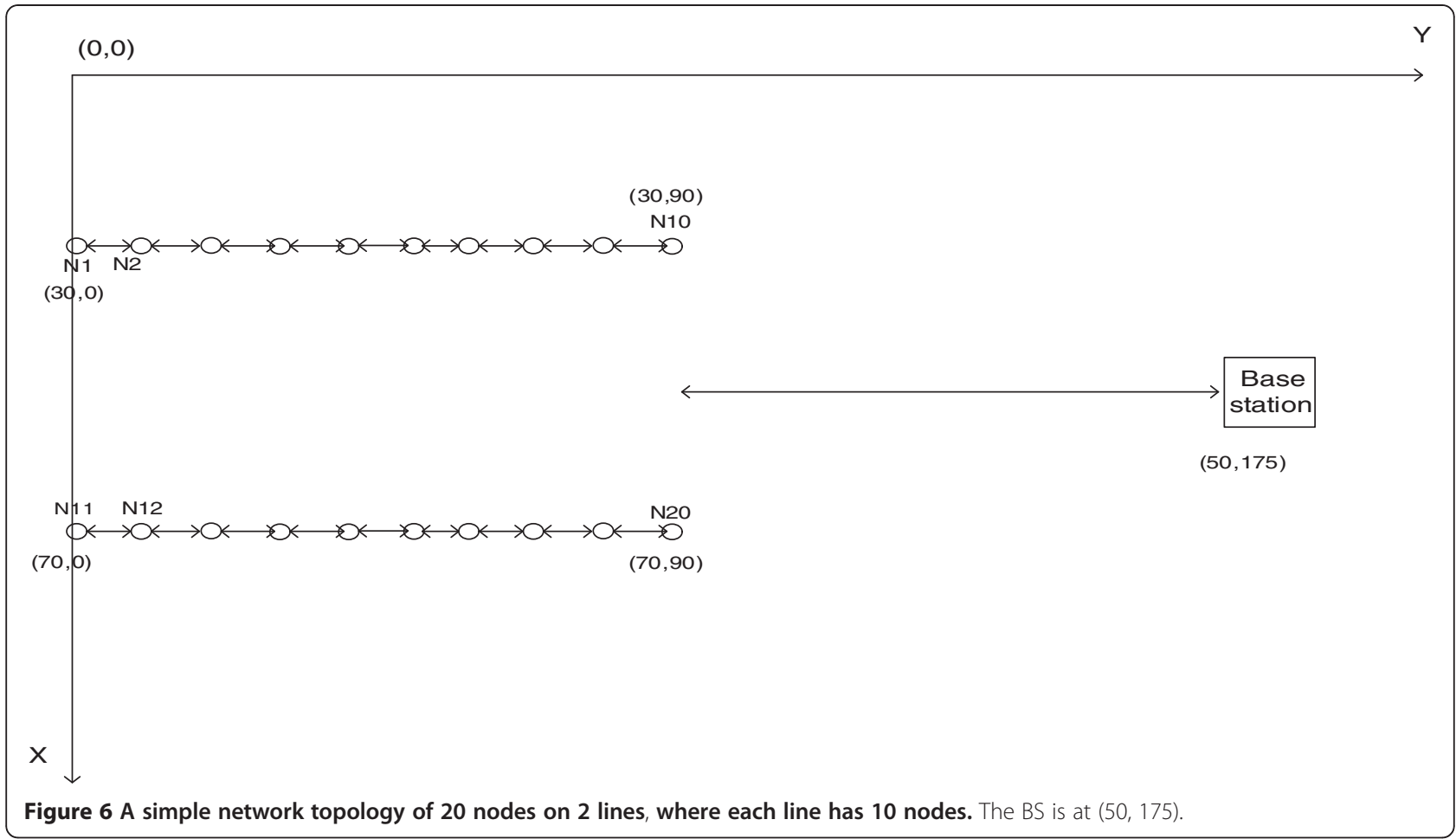

of (7) must be smaller than that of (8). This result is verified by using the ILP solver in [8]. The optimal solution of Simple problem (1) is 6 while the optimal solution of Simple problem (2) is 8 .

This theorem is important because in many cases, this is very hard to calculate $O_{1}$. One of the reasons is that working out all coefficients $c_{j}^{i}$ is impossible. Based on the theory, we know that $O_{2}$ can be an upper bound of $O_{1}$, or all the feasible solutions of Problem 1 are bounded by $\mathrm{O}_{2}$.

Theorem 2: Given a clustering sensor network with $k$ $\mathrm{CHs}$, connection from non- $\mathrm{CH}$ nodes to the closest $\mathrm{CH}$ node of the $k \mathrm{CHs}$ provides the optimal lifetime for the clustering network.

In more detail, we are given a set of $n$ sensors located in two-dimensional space $R^{2}$. Let us define $S$ as the set of ways to select $k \mathrm{CHs}$ in the given set of $n$ sensors. If every $\mathrm{CH}$ is different to the remaining $k-1 \mathrm{CHs}$, the number of elements in $S$ is $\left(\begin{array}{l}n \\ k\end{array}\right)$. However, in the theorem, some $\mathrm{CHs}$ might be the same and these same $\mathrm{CHs}$ are considered as one $\mathrm{CH}$. Therefore, the number of elements in $S$ is $n^{k}$ elements. Let us define $s_{n}^{k}(i)$ as the

Table 4 The average energy dissipated (units) per round over the number of $\mathrm{CHs}$

\begin{tabular}{llll}
\hline & $\mathbf{1} \mathbf{C H}$ & 2 CHs & 3 CHs \\
\hline Energy per round (units) & 65933 & 62016 & 69560 \\
\hline
\end{tabular}

$i$ th element in $S$ where $i$ in $\left(1 \ldots n^{k}\right)$. Let us define $c_{i}^{j}$ as the energy usage of Node $j$ consumes, when the $i$ th element in $S$ is selected as the CHs. Let us define $n_{i}$ as the number of rounds, which the $i$ th element in $S$ is selected as the CHs. Let us define $E_{j}$ as the initial energy of Node $j$ and $O$ as the optimal solution of the following ILP problem:

Maximize:

$$
\sum_{i=1}^{n^{k}} n_{i}
$$

Subject to:

$$
\sum_{i=1}^{n^{k}} n_{i} c_{i}^{j} \leq E_{j}: \forall j \in[1 \ldots n] n_{i} \in Z^{+}: \forall i \in\left[1 \ldots n^{k}\right]
$$

The energy $c_{i}^{j}$ is equal to the energy dissipation of Node $j$ to send a unit of data to the closest sensor node in the $i$ th element in $S$. Then, $O$ is the optimal lifetime for the sensor network with $k$ CHs.

Proof: Let us denote $c^{\prime j}{ }_{i}$ as the energy usage in any arbitrary way to send a unit of data from sensor node $j$ to the $i$ th element in $S, \forall i \in S, \forall j \in[1 \ldots n]$. The optimum selection of $\mathrm{CHs}$ of $S$ is found by solving the mixed integer programming (MIP) problem below: 


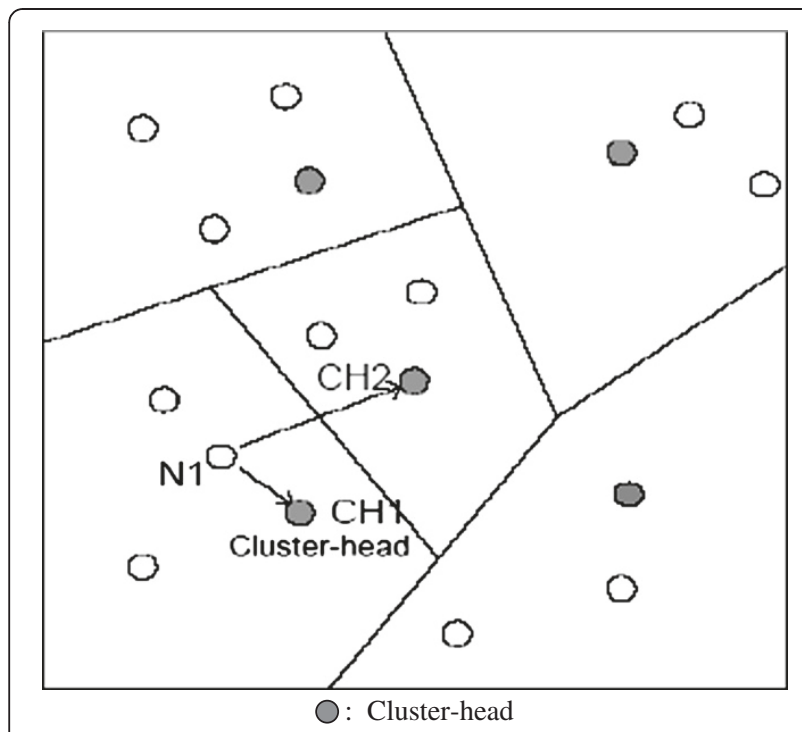

Figure 7 Connection from Node 1 to any $\mathrm{CH}$ will dissipate more energy than connection to $\mathrm{CH} 1$ (the closest $\mathrm{CH}$ of Node 1).

Maximize:

$$
\sum_{i=1}^{n^{k}} n_{i}
$$

Subject to:

$$
\sum_{i=1}^{n^{k}} n_{i} c_{i}^{\prime j} \leq E_{j}: \forall j \in[1 \ldots n] \quad n_{i} \in Z^{+}: \forall i \in\left[1 \ldots n^{k}\right]
$$

As $c_{i}^{\prime j} \geq c_{i}^{j} \forall i \in S, \forall j \in[1 \ldots n]$, since $c_{i}^{j}$ is equal to the energy dissipation of Node $j$ to send a unit of data to the closest sensor node in the $i$ th element in $S$, any optimum solution $O^{\prime}$ of (10) is smaller than the optimum solution $O$ obtained by (9) as Theorem 1 . This statement is illustrated in Figure 7. As the result, $O$ is the global optimum solution for maximizing the operation time with $k$ CHs.

\section{Calculation of coefficients for Problem (9)}

The energy coefficients $c_{i}^{j}$ of formulation (9) for a network of $n$ nodes with $k \mathrm{CHs}$ can be calculated as follows:

For every combination of $k \mathrm{CHs}$ from the $n$ nodes

For every node from the $n$ nodes

If (the node is a $\mathrm{CH}$ ) then

$$
c_{i}^{j}=d_{t o B S}^{2}
$$

else

$$
c_{i}^{j}=d_{t o C H}^{2}
$$

End of code

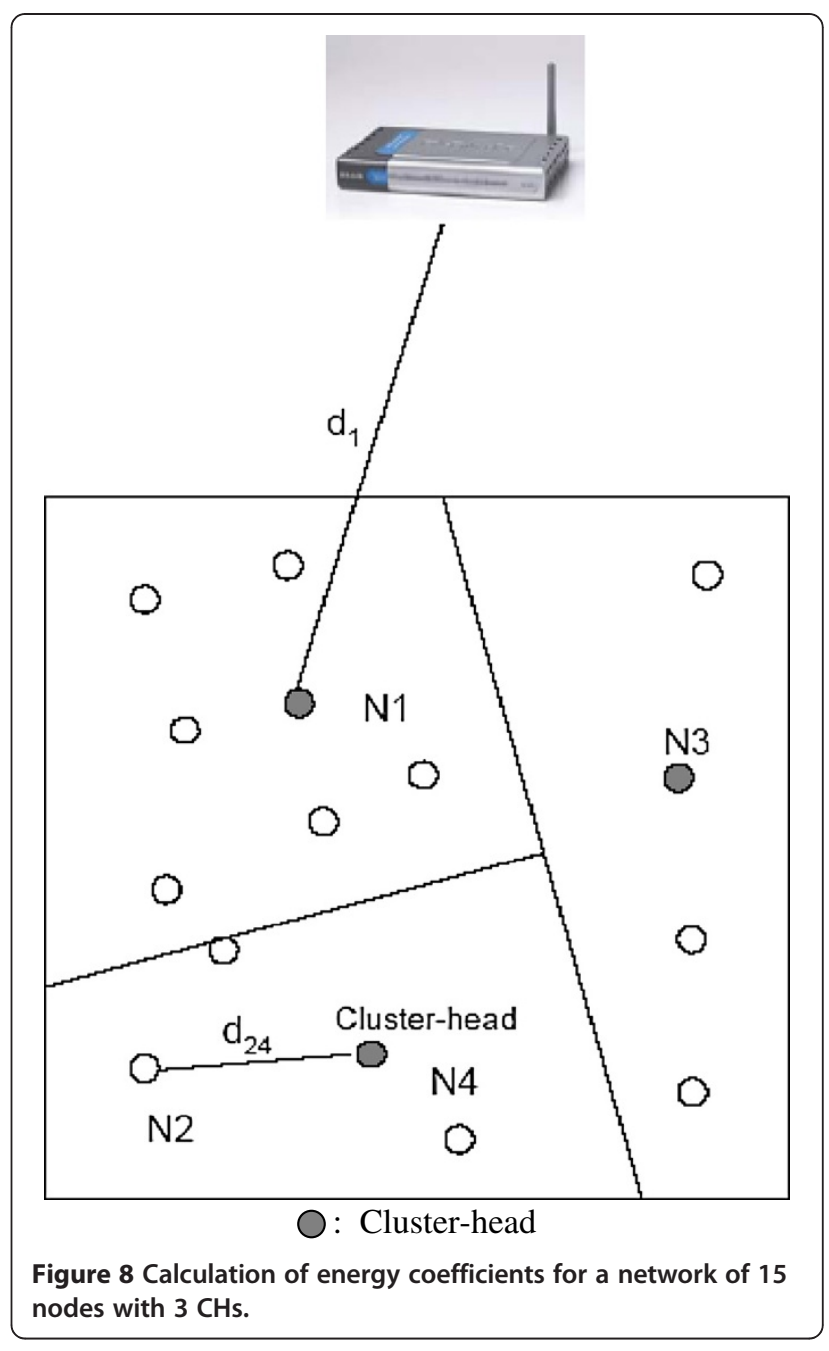

where $d_{\mathrm{toCH}}$ is the distance from the sensor node to the closest $\mathrm{CH}$ from the $k \mathrm{CHs}, d_{\mathrm{toBS}}$ is the distance from the sensor node to the BS.

Figure 8 shows that for the current selection of $k=3$ CHs and $n=15$ nodes, the energy coefficient of Node 2 is equal to $d_{24}^{2}$, and the energy coefficient of Node 1 is equal to $d_{1}^{2}$.

Theorem 3: The problem formulation in (9) provides the optimum solution for maximizing the operation time for any clustering network with the number of $\mathrm{CHs}$ smaller than or equal to $k$.

Proof:As stated in Theorem 2, S is the set of ways to select $k \mathrm{CHs}$ in the given set of $n$ sensors. In each

Table 5 The average energy dissipated (units) per round and the number of rounds over the number of $\mathrm{CHs}$

\begin{tabular}{llll}
\hline & $\mathbf{1} \mathbf{C H}$ & $\mathbf{2} \mathbf{C H s}$ & $\mathbf{3} \mathbf{C H s}$ \\
\hline Energy per round (units) & 65933 & 62016 & 69560 \\
Number of rounds & 332 & 377 & 364 \\
\hline
\end{tabular}


combination selection, some $\mathrm{CHs}$ might be identical and these identical $\mathrm{CHs}$ are considered as one $\mathrm{CH}$. In this case, the number of $\mathrm{CHs}$ is less than $k$. Therefore, any network of less than $k \mathrm{CHs}$ is a special element in $S$, where some $\mathrm{CHs}$ are the same.

It is of interest to know the optimum solution of the network topology in Figure 6. Every sensor node begins with 1 million units of energy and the above-mentioned simple energy model is used. Table 5 shows the optimum system lifetime versus the number of $\mathrm{CHs}$. The results show that the network achieves the optimum solution at the number of two $\mathrm{CHs}$.

It is also of interest to see the distribution of optimums CHs among the 20 sensor nodes in Figure 6. The distribution depends on the position of sensors. The energy model used is $d^{2}$ energy model (gamma $=2$ ).

Figure 9 shows the five pairs that are chosen as $\mathrm{CHs}$ most frequently. The results show that the pair of nodes $(7,17)$ is the most preferred CHs. This is due to the fact that the nodes are not very far from the BS as well as the rest of other nodes. As such, they can become intermediate $\mathrm{CHs}$ to deliver data to the BS. The five pairs are selected as $\mathrm{CHs}$ for $56 \%$ of the total number of rounds.

The same experiments are carried out on the same network over the "power 4" (gamma = 4) model. The model is given below:

$$
E(S)=\alpha d^{4}, E(D)=0, \text { for } \alpha>0
$$

where $S$ denotes a source node, Ddenotes a destination node, $E(S)$ is the energy usage of node $S$, and $d$ is the distance from $S$ to $D$. This formula states that the energy required to transmit a unit of data is proportional to the

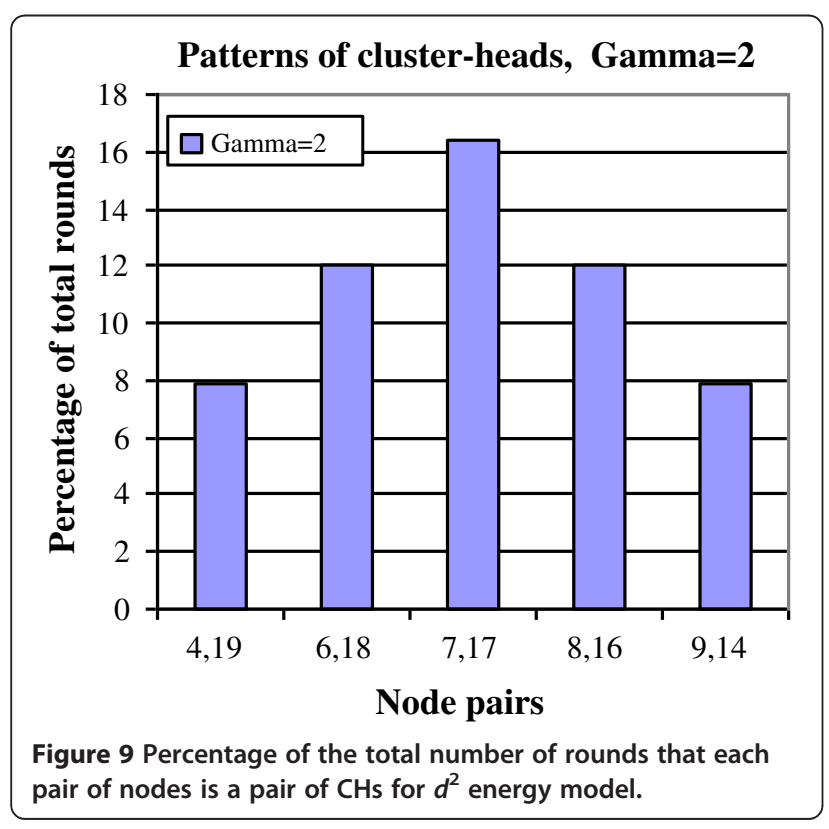

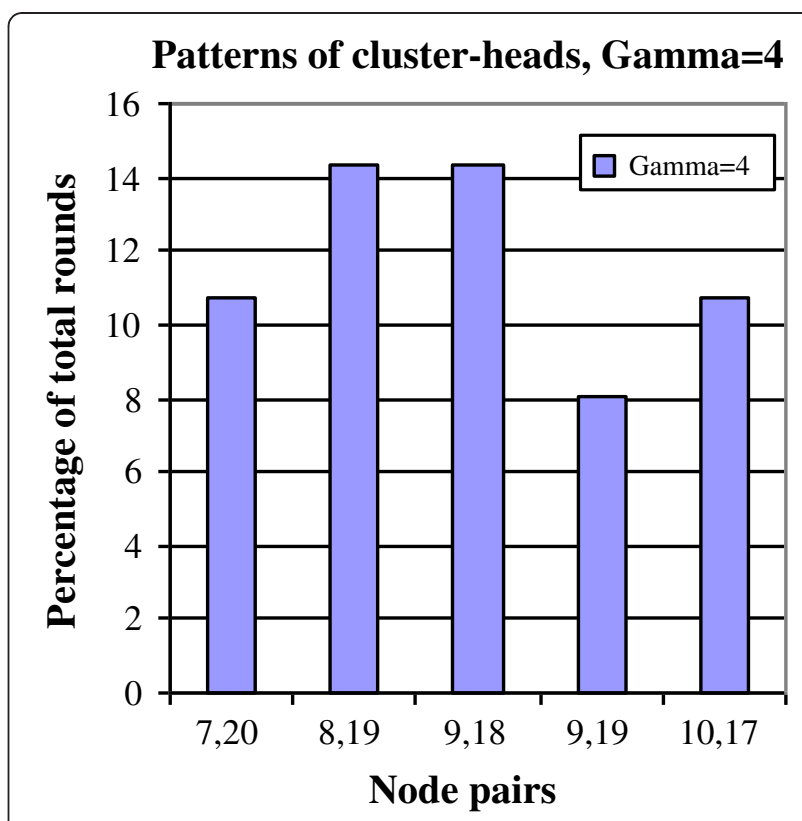

Figure 10 Percentage of the total number of rounds that each pair of nodes is a pair of $\mathrm{CHs}$ for $d^{4}$ energy model.

"power 4" of the distance to a destination, and there is no energy spent at the destination. For the rest of this section, $\alpha$ is set to 1 .

Figure 10 shows the simulation results when $\alpha$ is set to 1. Compared to the previous results, the $\mathrm{CHs}$ move closer to the BS. This is because when the "power 4" model is used, the energy of $\mathrm{CH}$ nodes is drained quickly. As such, the nodes need to be closer to the BS. The five pairs are selected as $\mathrm{CHs}$ for $58 \%$ of the total number of rounds.

\section{A simplified LEACH_C protocol (AVERA)}

As mentioned in the Section "Previous work in energy efficiency using cluster-based routing”, LEACH_C utilizes the BS for creating clusters. During the setup phase, the $\mathrm{BS}$ receives information about the location and the energy level of each node in the network. Using this information, the BS decides the number of $\mathrm{CHs}$ and configures the network into clusters. To do so, the BS computes the average energy of nodes in the network. Nodes that have energy storage below this average cannot become $\mathrm{CHs}$ for the next round. From the remaining possible $\mathrm{CH}$ nodes, the $\mathrm{BS}$ uses the SA algorithm to find the $k$ optimal $\mathrm{CHs}$. The selection problem is an NP-hard problem.

If the BS is also far away from main power sources and is energy-limited and processing-limited, it is impractical for the BS to run $\mathrm{LEACH}$ _C as it creates significant delay and requires significant computation. In this case, we modify LEACH_C algorithm by removing 
the SA algorithm process. In more details, our algorithm AVERA is implemented as below.

AVERA:

In every round, select $k \mathrm{CHs}$ randomly from $m$ sensor nodes that have their energy level above the average energy of all nodes.

\section{Given:}

$N$ : The number of sensor nodes indexed from 1 to $N$

$s$ : The current $\mathrm{CH}$ solution

$m$ : The number of sensor nodes that have energy above the average energy of all sensors

For every round of data transmission

$s=k$ sensors in Random $[1 \ldots m]$

Result: $s$ is the $\mathrm{CH}$ solution for the round obtained from the AVERA algorithm. (End of code)

\section{Simulation and comparison}

Most of previous work on WSN lifetime [1-5] used the energy consumption model and the energy dissipation parameters given in [9]. The data are kept the same in our experiments to make the comparison between our proposed algorithms and previous ones feasible. The power transmission coefficients for free space and multipath are given below.

$$
\begin{aligned}
& \varepsilon_{F S}=10 \mathrm{pJ} / \mathrm{b} / \mathrm{m}^{2} \\
& \varepsilon_{M P}=.0013 \mathrm{pJ} / \mathrm{b} / \mathrm{m}^{4}
\end{aligned}
$$

From the parameters, the output power of a transmitter over a distance $d$ is given by

$$
\begin{aligned}
& P_{\text {amp }}(d)=\left\{\varepsilon_{F S} k d^{2}, d<d_{o}\right. \\
& P_{\text {amp }}(d)=\left\{\varepsilon_{M P} k d^{4}, d>d_{o}\right.
\end{aligned}
$$

where $d_{o}$ is set to $82.6 \mathrm{~m}$. The value of $E_{\text {elec }}$ follows the experiments in [1,2,17-19] and is set to $50 \mathrm{~nJ} / \mathrm{bit}$.

In summary, the total transmission energy of a message of $k$ bits in sensor networks is calculated by

$$
\begin{aligned}
& E_{t}=k E_{\text {elec }}+\varepsilon_{\mathrm{FS}} k d^{2}, \text { when } d<d_{o} \\
& E_{t}=k E_{\text {elec }}+\varepsilon_{\mathrm{MP}} k d^{4}, \text { when } d>d_{o}
\end{aligned}
$$

and the reception energy is calculated by

$$
E_{r}=E_{\text {elec }} k
$$

where $E_{\text {elec }}, \varepsilon_{\mathrm{FS}}, \varepsilon_{\mathrm{MB}}$ and $d_{o}$ are given above.

First, the optimum number of $\mathrm{CHs}$ of these networks is studied. In the experiments, 100 random 80-node sensor networks are generated. Each node begins with $1 \mathrm{~J}$ of energy. The network settings for the simulations are given

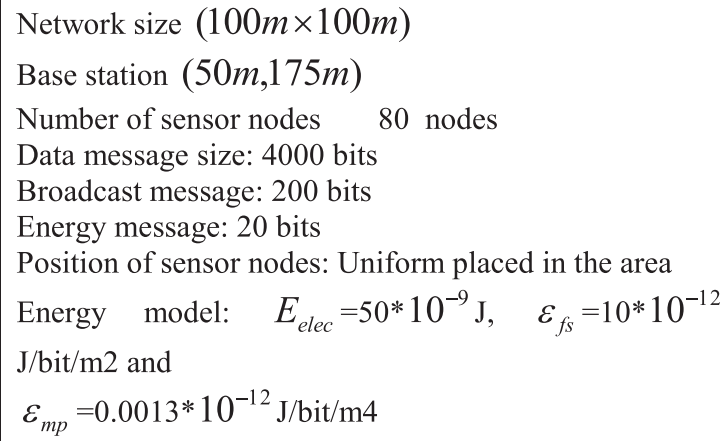

Figure 11 Average energy dissipation per round (units) over the number of $\mathrm{CHs}$.

below. The sensor positions and the BS position are defined as below . This is the same settings used in $[1-5,9,18,19]$.

Network size $(100 m \times 100 m)$

Base station $(50 m, 175 m)$

Number of sensor nodes 100 nodes

Data message size: 4000 bits

Broadcast message: 200 bits

Energy message: 20 bits

Position of sensor nodes: Uniform placed in the area

Energy model: $E_{\text {elec }}=50^{*} 10^{-9} \mathrm{~J}, \varepsilon_{f s}=10^{*} 10^{-12} \mathrm{~J} / \mathrm{bit} /$ $\mathrm{m}^{2}$ and $\varepsilon_{m p}=0.0013^{*} 10^{-12} \mathrm{~J} / \mathrm{bit} / \mathrm{m}^{4}$

During the sensor operation, every sensor node sends data periodically to the BS. A round of data transmission is defined as the duration of time to send a unit of data (4000 bits) to the BS. Each round consists of a setup and a transmission phase. In the setup phase, the network is divided into clusters and nodes negotiate to nominate $\mathrm{CHs}$ for the round. In the LEACH_C and AVERA protocols, each node sends its energy level message to the BS (20 bits). The BS decides the $\mathrm{CHs}$ for the round and sends a broadcast message (200 bits) about the decision for the round to all sensor networks.

In the transmission phase, the elected $\mathrm{CH}$ collects all data from nodes in its cluster and forwards the data to a BS. After each round, every sensor node loses an

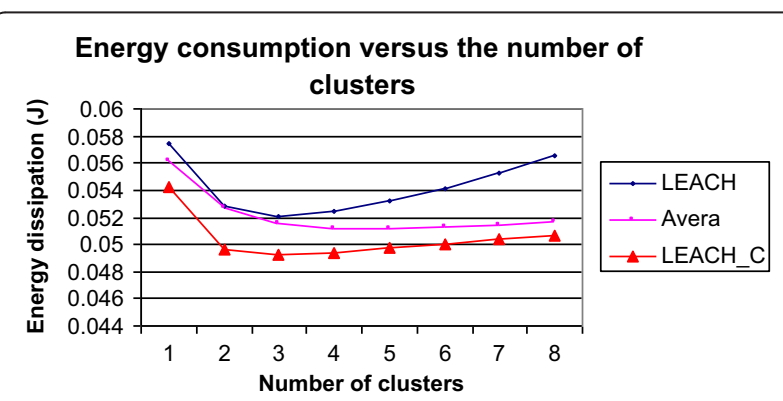

Figure 12 Ratio of the number of rounds between RE, LEACH, and the optimum solution. 
amount of energy for the data transmission in the round. The amount depends on the distance from the sensor to its $\mathrm{CH}$ or to the BS. The lifetime of sensor networks is measured as the total number of rounds sending data to the BS until the first node is off.

LEACH, LEACH_C, and AVERA are used over 100 network topologies while varying the number of $\mathrm{CHs}$ from 1 to 8 , and the system lifetime and the energy dissipation per round are recorded for these numbers of CHs.

Figure 11 shows that the energy dissipation per round is minimized for LEACH, LEACH_C, and AVERA at the number of $\mathrm{CHs}$ from 3 to 4 . The result agrees well with the analytical model and the results are presented in $[1,2,17]$.

\section{Validation of the analytical model}

In this section, the performance of $\mathrm{LEACH}, \mathrm{LEACH} C$, and AVERA and the optimum solution from the analytical model is verified. The number of $\mathrm{CHs}$ is set to three in all methods. All methods are run over the above 100 random 80-node network topologies and the ratio between the lifetime of the three protocols and the optimum are recorded. For the calculation of the optimum solution, we use the GNU Linear Programming Kit (GLPK) and the MIP solver. GLPK is a free GNU LP software package for solving large-scale LP, MIP [8].

GLPK provides two methods to solve LP and MIP problems:

(1) Create a problem in $C$ programming language that calls GLPK API routines

(2) Create a problem in a text editor and use a standalone LP/MIP solver to solve it.

We use method 2 to calculate the optimum solution. Figure 12 shows that both AVERA and LEACH_C perform very closely to the optimum solution while $\mathrm{LEACH}$ is only $70 \%$ of the optimum solution.

The computation time for all three protocols is also recorded on the 100 network topologies. The computational time for LEACH, AVERA, and LEACH_C are 1.6,2.5, and173.2 s, respectively. This shows that the new protocol AVERA provides a reasonably good operation time while guarantees less processing from the BS.

\section{Conclusion}

This article has presented some energy-efficient clusterbased routing protocols. In sensor networks, BSs only require a summary of the events occurring in their environment, rather than the sensor node's individual data. To exploit the function of the sensor networks, sensor nodes are grouped into small clusters so that $\mathrm{CH}$ nodes can collect the data of all nodes in their cluster and perform aggregation into a single message before sending the message to the BS. Since all sensor nodes are energy-limited, $\mathrm{CH}$ positions should be reallocated among all nodes in the network to extend the network lifetime. The determination of adaptive clusters is not an easy problem. We start by analyzing simple networks with one $\mathrm{CH}$ first to be able to obtain an effective solution for the problem. Then the model is extended to networks with multiple $\mathrm{CHs}$.

Heuristic algorithms are also proposed to solve the problem. Simulation results show that LEACH solution performs quite far from the optimum solution as it does not directly work on the remaining energy of all sensor nodes. At the same time, both AVERA and LEACH_C solutions perform very closely to the optimum solution. Note that the computational time for AVERA is also $1.4 \%$ of $\mathrm{LEACH}$ C.

\section{Competing interests}

The authors declare that they have no competing interests.

\section{Acknowledgment}

This research is carried out under the frame work of the Project number 102.04-2012.35 funded by the Vietnamese National Foundation for Science and Technology Development (NAFOSTED).

\section{Author details}

IInternational School, Vietnam National University, 144 Xuan Thuy, Cau Giay, Hanoi, Vietnam. ${ }^{2}$ Faculty of Electronics and Telecommunications, Hanoi University of Science and Technology, Dai Co Viet Str. 1, Hanoi, Vietnam.

Received: 13 September 2011 Accepted: 26 October 2012 Published: 21 November 2012

\section{References}

1. WB Heinzelman, AP Chandrakasan, H Balakrishnan, Energy-efficient communication protocol for wireless microsensor networks, in 33rd Hawail International Conference Systems Sciences, 2000, p. 10. Vol.2. ISBN: 0-76950493-0

2. WB Heinzelman, AP Chandrakasan, H Balakrishnan, An application specific protocol architecture for wireless microsensor networks. IEEE Trans. Wirel. Commun 1(4), 660-670 (2002)

3. I Saha Misra, S Dolui, A Das, Enhanced-efficient adaptive clustering protocol for distributed sensor networks, in IEEE ICON, 2005, p. 6. doi:10.1109/ ICON.2005.1635525. Vol.1, ISBN:1-4244-0000-7

4. J Zhang, B Huang, L Tu, F Zhang, A cluster-based energy-efficient scheme for sensor networks, in The Sixth International Conference on Parallel and Distributed Computing, Applications and Technologies (PDCAT'05), 2005, pp. 191-195. doi:10.1109/PDCAT.2005.3. ISBN: 0-7695-2405-2

5. R Chang, C Kuo, An energy efficient routing mechanism for wireless sensor networks, in The 20th International Conference on Advanced Information Networking and Applications (AINA'06), 2006. Vol.2, p. 5. doi:10.1109/ AINA.2006.86. ISSN: 1550-445X; ISBN: 0-7695-2466-4

6. JN Al-Karaki, AE Kamal, Routing techniques in wireless sensor networks: a survey. IEEE Wirel. Commun. 11(6), 6-28 (2004)

7. J Deng, YS Han, W Heinzelman, Balanced-energy sleep scheduling scheme for high density cluster-based sensor networks. Comput. Commun. 28 1631-1642 (2005)

8. GLPK programming, 2011. http://www.gnu.org/software/glpk/

9. T Nguyen Thanh, Energy-efficient routing algorithms in wireless sensor networks. PhD thesis (Monash University, Australia, 2008)

10. L Guo, Y Xie, C Yang, Z Jing, Improvement on LEACH by combining adaptive cluster head election and two-hop transmission, in 9th International Conference on Machine Learning and Cybernetics, 2010, pp. 1678-1683 
11. T Qiang, W Bingwen, W Zhicheng, MS-LEACH: a routing protocol combining multihop transmission and single-hop transmission, in PacificAsia Conference on Circuits, Communications and Systems, 2009, pp. 107-110

12. M Farooq, AB Dogar, GA Shah, MR-LEACH: multi-hop routing with low energy adaptive clustering hierarchy, in 4th International Conference on Sensor Technologies and Applications, 2010, pp. 262-268

13. J Chen, $\mathrm{H}$ Shen, MELEACH-L: more energy efficient LEACH for large scale WSNs, in Wireless Communications, Networking and Mobile Computing, 2008, pp. 1-4

14. SG Nash, A Sofer, Linear and Nonlinear Programming (McGraw-Hill, New York, 1996)

15. T Kanungo, DM Mount, NS Netanyahu, A local search approximation algorithm for k-means clustering, in The Eighteenth Annual Symposium on Computational geometry, 2002. Volume 28, Issues 2-3, pp. 89-112

16. S Lindsey, C Raghavendra, Power-efficient gathering in sensor information systems, in IEEE Aerospace Conference, 2002. Vol.3, p. 3-1125-3-1130. doi:10.1109/AERO.2002.1035242.0-7803-7231-X

17. WB Heinzelman, Application-specific protocol architectures for wireless networks. PhD dissertation (Massachusetts Institute of Technology, 2000)

18. T Nguyen Thanh, $V$ Phan Cong, The Energy-Aware Operational Time of Wireless Ad-hoc Sensor Networks. ACM/Springer Mobile Networks and Applications (MONET) Journal, Volume 17, August, 2012. doi:10.1007/s11036012-0403-1

19. T Nguyen Thanh, Heuristic Energy-Efficient Routing Solutions to Extend the Lifetime of Wireless Ad-Hoc Sensor Networks (Springer, LNCS 7197, 2012), pp. 487-497. ISBN: 978-3-642-28489-2

doi:10.1186/1687-1499-2012-348

Cite this article as: Nguyen and Nguyen: Optimizing the operating time of wireless sensor network. EURASIP Journal on Wireless Communications and Networking 2012 2012:348.

\section{Submit your manuscript to a SpringerOpen ${ }^{\circ}$ journal and benefit from:}

- Convenient online submission

- Rigorous peer review

- Immediate publication on acceptance

- Open access: articles freely available online

- High visibility within the field

- Retaining the copyright to your article

Submit your next manuscript at $\gg$ springeropen.com 